\title{
An elegant technology for ultrasensitive impedimetric and voltammetric determination of cholestanol based on a novel molecularly imprinted electrochemical sensor
}

\author{
Ali R. Jalalvand ${ }^{\mathrm{a}, *}$, Mohammad Mahdi Zangeneh ${ }^{\mathrm{c}, \mathrm{d}}$, Faramarz Jalili ${ }^{\mathrm{a}}$, Shokoufeh Soleimani ${ }^{\mathrm{a}}$, \\ Jose Manuel Díaz-Cruz ${ }^{\mathrm{b}}$, \\ ${ }^{a}$ Research Center of Oils and Fats, Research Institute for Health Technology, Kermanshah University of Medical Sciences, Kermanshah, \\ Iran \\ ${ }^{\mathrm{b}}$ Departament d'Enginyeria Química i Química Analítica, Facultat de Química, Universitat de Barcelona, Martí i Franques 1-11, E- \\ 8028 Barcelona, Spain \\ ${ }^{\mathrm{c}}$ Department of Clinical Sciences, Faculty of Veterinary Medicine, Razi University, Kermanshah, Iran \\ ${ }^{\mathrm{d}}$ Biotechnology and Medicinal Plants Research Center, Ilam University of Medical Sciences, Ilam, Iran \\ *Corresponding Author: Tel.: +988334302345, Fax: +988334279745 \\ E-mail Address: ali.jalalvand1984@gmail.com (A.-R. Jalalvand)
}

\begin{abstract}
In this work, a novel molecularly imprinted electrochemical sensor (MIES) has been fabricated based on electropolymerization of a molecularly imprinted polymer (MIP) onto a glassy carbon electrode (GCE) modified with gold-palladium alloy nanoparticles (AuPd NPs)/polydopamine film (PDA)/multiwalled carbon nanotubeschitosan-ionic liquid (MWCNTs-CS-IL) for voltammetric and impedimetric determination of cholestanol (CHO). Modifications applied to the bare GCE formed an excellent biocompatible composite film which was able to selectively detect $\mathrm{CHO}$ molecules. Modifications applied to the bare GCE were characterized by scanning electron microscopy (SEM), cyclic voltammetry (CV) and electrochemical impedance spectroscopy (SEM). Under optimal experimental conditions, the sensor was able to detect $\mathrm{CHO}$ in the range of $0.1-60 \mathrm{pM}$ and $1-50 \mathrm{pM}$ by EIS and DPV, respectively. Moreover, the sensor showed high sensitivity, selectivity, repeatability, reproducibility, low interference and good stability towards $\mathrm{CHO}$ determination. Our records confirmed that the sensor was successfully able to the analysis real samples for determination of $\mathrm{CHO}$.
\end{abstract}

Keywords: Cholestanol; Molecularly imprinted polymer; Electrochemical sensor. 


\section{Introduction}

Increasing cholestanol (CHO) levels in the blood serum is a biochemical marker for diagnosis of cerebrotendinous xanthomatosis (CTX) [1,2] and liver and biliary tract diseases [3-6]. CHO determination is also used for following-up of patients with cirrhosis, primary biliary cirrhosis, or after liver transplantation [4-6]. Therefore, determination of $\mathrm{CHO}$ is important from clinical point of view. Different analytical methods including gas chromatography (GC) [7,8], capillary GC [3,6,9], GC-mass spectrometry (GC-MS) [10], high-performance liquid chromatography (HPLC) with fluorescence detection [11-13], and HPLC with UV detection [14-17] have been reported for determination of $\mathrm{CHO}$. These methods require complex procedures for precipitation of lipoprotein fractions which suffer from low specificity, instability of reagents and high cost. Therefore, developing novel methods which are able to sensitive, selective, stabile, repeatable, reproducible and low-cost determination of CHO are highly demanded.

Molecularly imprinted electrochemical sensors (MIESs) use a wholly synthetic recognition element which makes them to be less expensive devices than chemo/biosensors. The MIESs are good devices with high sensitivity, good selectivity and fast response towards small molecules and biomacromolecules [18,19]. The MIESs are benefited from electrochemical polymerization which help them to have a controllable polymer film on their surface which cannot be accessible by conventional synthetic methods. A very important thing in fabrication of MIESs is related to the improvement of the immobilization of molecularly imprinted polymer (MIP) onto the electrode surface and high affinity for the target analyte [20,21]. Therefore, there are many reports on different strategies performed for improving immobilization of the MIP and its affinity towards target analyte [22-25]. Herein, we are going to develop a novel and biocompatible MIES to capture CHO biomacromolecules in which bioinspired polydopamine (PDA) films will be used to achieve better imprinted effect and faster preparation than self-assembly monolayers. 
The PDA is a suitable surface-adherent material for multifunctional coatings of membrane surfaces. Dopamine is able to self-polymerization in alkaline condition to form a strong and biocompatible layer on the substrate on which it is grown [26]. Therefore, the PDA is able to immobilize multiwalled carbon nanotubes (MWCNTs) onto the surface of a glassy carbon electrode (GCE). Furthermore, the PDA layer has catechol and amino groups and catechols can react with thiols and amines, and aminos are able to react with hydroxyls and carboxyls [27]. Therefore, the PDA is able to interact with aminothiophenol (ATP) molecular imprinting films by intermolecular forces. The PDA film has a cross-linked structure on which could generate stable threedimensional imprinting sites and capture more template $\mathrm{CHO}$ molecules. Furthermore, binding ability of catechols supports metallization in situ which could stabilize metallic nanoparticles [28]. The MWCNTs due to their remarkable electrical, chemical, mechanical and structural properties are very important in developing MIESs [29]. Ionic liquids (ILs) have excellent properties such as stability, high electrical conductivity and low vapor pressure [30]. Because of $\pi-\pi$ or cation- $\pi$ interaction between MWCNTs and IL, the MWCNTs-IL composite has obtained potential applications in construction of electrochemical sensors and biosensors [31]. Chitosan (CS) as a natural, biocompatible, nontoxic and water permeable biopolymer has a good film forming ability which is frequently used to fabricate electrochemical sensors and biosensors [32-34]. Bimetallic alloy nanoparticles (NPs) are widely used in fabrication of electrochemical sensors and biosensors for increasing surface area, enhancing electrocatalytic activity and promoting electron transfer in comparison with their corresponding monometallic counterparts [35]. Sensitivity of an imprinted sensor is affected by the amount of effective imprinted sites on its surface which could be increased by the presence of NPs due to their large specific surface area, good biocompatibility and high conductivity.

In fabrication of electrochemical sensors, it is usual to use a GCE as the platform of the sensor which can be modified by different modifiers to increase its sensitivity and selectivity and sometimes the GCE response is assisted by computerized programs to improve its performance [36-54]. Therefore, in this work we are going to fabricate a novel MIES based on electropolymerization of ATP onto AuPd NPs/PDA/MWCNTs-CS-IL/GCE. Here, AuPd NPs could act as crosslinkers to form polymeric networks by Au-S in MIP preparation. After testing 
performance of the developed MIES towards determination of CHO in synthetic samples, it will be applied to the determination of $\mathrm{CHO}$ in real samples. Schematic representation of the procedure used in this study to electrochemical sensing of $\mathrm{CHO}$ is shown in Scheme 1.

\section{Scheme 1}

\section{Experimental}

\subsection{Chemicals and solutions}

CHO, dopamine, ATP, CS, $\mathrm{CHO}, \quad \mathrm{LiClO}_{4}, \quad \mathrm{HClO}_{4}$, ethanol, 1-ethyl-3-methylimidazolium bis(trifluoromethylsulfonyl)imide (IL), potassium ferrocyanide, potassium ferriccyanide, $\mathrm{HAuCl}_{4}$, palladium (II) chloride $\left(\mathrm{PdCl}_{2}\right)$, dimethylformamide (DMF), acetic acid, sodium phosphate monobasic $\left(\mathrm{NaH}_{2} \mathrm{PO}_{4}\right)$, sodium phosphate dibasic $\left(\mathrm{Na}_{2} \mathrm{HPO}_{4}\right), \mathrm{H}_{3} \mathrm{PO}_{4}, \mathrm{Na}_{2} \mathrm{SO}_{4}$, MWCNTs and $\mathrm{NaOH}$ were purchased from Sigma. The other chemicals used in this work were purchased from well-known sources and used without further purifications. A phosphate buffer solution (PBS) with a concentration of $0.05 \mathrm{M}$ was prepared from $\mathrm{NaH}_{2} \mathrm{PO}_{4}$ and $\mathrm{Na}_{2} \mathrm{HPO}_{4}$ and its $\mathrm{pH}$ was adjusted at 7.4 by $\mathrm{H}_{3} \mathrm{PO}_{4}$ and $\mathrm{NaOH}$. A stock solution of $\mathrm{CHO}$ with a concentration of $0.1 \mathrm{M}$ was prepared in ethanol and kept frozen at $-20^{\circ} \mathrm{C} .1 \mathrm{mg}$ MWCNTs was mixed with $1 \mathrm{~mL}$ DMF and $50 \mu \mathrm{L}$ IL was added to it and ultrasonicated for 1 hour. A solution containing $\mathrm{PdCl}_{2} 0.5 \mathrm{mM}$ and $\mathrm{HAuCl}_{4} 0.5 \mathrm{mM}$ was prepared in $\mathrm{Na}_{2} \mathrm{SO}_{4} 0.1 \mathrm{mM}$ which was used for electrodeposition of AuPd NPs onto the surface of the sensor. $0.2 \mathrm{mg}$ CS was added to $0.5 \mathrm{~mL}$ acetic acid and ultrasonicated for 1 hour. To prepare MWCNTs-CS-IL, $0.5 \mathrm{~mL}$ MWCNTsIL was added to $0.5 \mathrm{~mL} \mathrm{CS}$ and ultrasonicated for $15 \mathrm{~min}$. A probe solution consisting of $5 \mathrm{mM} \mathrm{K}_{3} \mathrm{Fe}(\mathrm{CN})_{6}$ and 5 $\mathrm{mM} \mathrm{K} \mathrm{Ke}_{4}(\mathrm{CN})_{6}$ was prepared in $0.1 \mathrm{M} \mathrm{KCl}$.

\subsection{Instruments and software}

An Autolab PGSTAT302N-high performance under controlling by the NOVA 2.1.2 software was used to record electrochemical data. All the electrochemical experiments were performed in an electrochemical cell in which an $\mathrm{Ag} / \mathrm{AgCl}$, a Pt wire and a bare or modified GCE were acted as reference, counter and working electrode, respectively. $\mathrm{pH}$ adjustments were performed by an ELMEIRON pH meter (CP-411). A KYKY-EM 3200 scanning electron microscope was used to record scanning electron microscopic (SEM) images. Energy dispersive X-ray spectroscopy (EDS) were performed for elemental analysis by an EDS-integrated Hitachi S-4800. All the 
computations required in this work were performed on a DELL XPS laptop (L502X) with a Windows 10 as its operating system.

\subsection{Preparation of the sensor}

Prior to the modification of the bare GCE, it was well-polished on a silky pad impregnated with alumina slurry and rinsed with doubly distilled water (DDW) and dried by a hair dryer. The cleaned GCE was covered until using time and cleaning of the GCE was performed according to the described procedure whenever was needed. $7 \mu \mathrm{L}$ MWCNTs-CS-IL was drop-casted onto the surface of the GCE and left to be died at room temperature to fabricate MWCNTs-CS-IL/GCE. Then, the MWCNTs-CS-IL/GCE was immersed into a PBS (0.05 M, pH 7) containing 10

$\mathrm{mg} \mathrm{mL} \mathrm{m}^{-1}$ dopamine to electropolymerize dopamine on the electrode surface and formation of PDA by cyclic voltammetry $(\mathrm{CV})$ under scanning potential between -0.4 and $+1.5 \mathrm{~V}$ at $50 \mathrm{mV} \mathrm{s}^{-1}$ for 8 cycles. Electrochemical deposition of AuPd NPs onto the surface of PDA/MWCNTs-CS-IL/GCE was performed by immersing it into a solution containing $\mathrm{PdCl}_{2} 0.5 \mathrm{mM}$ and $\mathrm{HAuCl}_{4} 0.5 \mathrm{mM}$ prepared in $\mathrm{Na}_{2} \mathrm{SO}_{4} 0.1 \mathrm{mM}$ and potential was scanned from 0 to -1 for 10 cycles and afterwards, the electrode was rinsed with DDW and left to be dried at room temperature. Then, the AuPd NPs/PDA/MWCNTs-CS-IL/GCE was immersed into an ethanol solution containing $25 \mathrm{mM}$ ATP, $50 \mathrm{mM} \mathrm{CHO}$ and $0.1 \mathrm{M} \mathrm{LiClO}_{4}$ and electropolymerization was performed by scanning potential in the range of $-0.5-0.8 \mathrm{~V}$ for 6 cycles with a scan rate of $50 \mathrm{mV} \mathrm{s}^{-1}$. Afterwards, for removing CHO template molecules, the MIP/AuPd NPs/PDA/MWCNTs-CS-IL/GCE was immersed into an ethanol solution containing 0.5 $\mathrm{M} \mathrm{HCl}$ and treated at a constant potential of $-0.1 \mathrm{~V}$ for $40 \mathrm{~s}$ and finally, the MIP/AuPd NPs/PDA/MWCNTs-CSIL/GCE was rinsed with DDW and dried by nitrogen. A nonimprinted sensor (NIP/AuPd NPs/PDA/MWCNTsCS-IL/GCE) was fabricated as a control under the same procedure without CHO template molecules.

\section{Results and discussion}

\subsection{Characterization of the modifications applied to the GCE}


Characterization of the modifications applied to the bare GCE is necessary to confirm successfulness of the fabrication procedure. Therefore, we are going to use some characterization techniques to confirm fabrication process applied to the bare GCE which will be expanded in details in next sections.

\subsubsection{Electrochemical characterization}

Electrochemical impedance spectroscopy (EIS) and CV are two well-known electrochemical techniques which have been frequently used to characterize modifications.

An EIS curve has a semicircle portion whose diameter is correlated with charge transfer resistance $\left(R_{\mathrm{ct}}\right)$ at the electrode surface. Modification of the electrode surface affects the $R_{\mathrm{ct}}$ which can be used to characterize modifications applied to the electrode surface. In order to characterize modifications by the EIS method, bare or modified GCEs were used as working electrode immersed into the probe solution consisting of $5 \mathrm{mM} \mathrm{K} \mathrm{K}_{3} \mathrm{Fe}(\mathrm{CN})_{6}$ and $5 \mathrm{mM} \mathrm{K}_{4} \mathrm{Fe}(\mathrm{CN})_{6}$ prepared in $0.1 \mathrm{M} \mathrm{KCl}$ and the results are shown in Fig. 1A. As can be seen, the bare GCE has an EIS curve (curve $a$ ) with a diameter of $684 \Omega$ which was decreased $(310 \Omega$, curve $b$ ) after its modification by MWCNTs-CS-IL which confirmed that faster charge transfer was occurred at the surface of MWCNTs-CSIL/GCE than bare GCE due to the presence of MWCNTs-CS-IL. MWCNTs due their nanostructure have a larger surface area and the IL due to its high ionic conductivity can increase the conductivity of the modifier layer in combination with each other. Integration CS with MWCNTs and IL can help to the stability of the composite film. By the formation of PDA at the electrode surface, the radius of the semicircle was increased (curve $c$ ) which may be related to the blocking the surface of electrode and hindering charge transfer in some extent. Electrodeposition of AuPd NPs onto the electrode surface caused decreasing of $R_{\mathrm{ct}}$ (curve $d$ ) due to the formation of a porous structure which increased the pathways for reaching the molecule probes to the electrode surface. Our records showed that the radius of the EIS curves of MIP/AuPd NPs/PDA/MWCNTs-CS-IL/GCE and NIP/AuPd NPs/PDA/MWCNTs-CS-IL/GCE was significantly increased (curves $e$ and $f$ ) which may be related to the large obstruction effect of the ATP films. It was important to see lower impedance for the MIP modified electrode than the NIP modified electrode which may be related to the large numbers of the imprinted cavities of $\mathrm{CHO}$ in the MIP films enhanced the diffusion of the redox probe and electron transfer. 
Fig. 1

Further electrochemical characterization of the modifications used to fabricate the MIES in this work was performed by the use of $\mathrm{CV}$ and the results are shown in Fig. 1B. As can be seen, the bare GCE showed a reversible response (curve $a$ ) which was strongly enhanced by the presence of MWCNTs-CS-IL at the electrode surface (curve $b$ ). The PDA blocked the electrode surface which caused a poorer response as shown by curve $c$. AuPd NPs/PDA/MWCNTs-CS-IL/GCE showed an enhanced response (curve $d$ ) which was related to the increase of surface roughness and effective surface area by the presence of AuPd NPs. The CVs of MIP/AuPd NPs/PDA/MWCNTs-CS-IL/GCE and NIP/AuPd NPs/PDA/MWCNTs-CS-IL/GCE are shown by curves $e$ and $f$, respectively. As can be seen, the MIP modified electrode showed a pair of redox peaks because of the presence of cavities in the MIP films which enabled probe molecules to diffuse through the MIP films and to take place redox reaction on the modified electrode while the presence of NIP at the electrode surface caused no redox peaks because of blocking electron transfer by the NIP films.

\subsubsection{Microscopic characterization and elemental analysis}

The SEM is a useful technique which can help us to monitor the electrode surface and modifications applied to it. Therefore, we have used it to monitor modifications applied to the bare GCE. The SEM images taken from the surface of MWCNTs-CS-IL/GCE, PDA/MWCNTs-CS-IL/GCE，AuPd NPs/PDA/MWCNTs-CS-IL/GCE and MIP/AuPd NPs/PDA/MWCNTs-CS-IL/GCE are shown in Fig. 2A-D, respectively. Tubes of the CNTs have twined around each other and integration of them with CS and IL has formed a layer on the GCE surface. Polymerization of dopamine concealed the MWCNTs-CS-IL by forming a new layer on its surface and further modification of the electrode by electrodeposition of AuPd NPs on its surface helped us to obtain a well-decorated surface which can be clearly seen in Fig. 2C. Formation of the MIP onto the electrode surface as the final layer of the sensor covered its surface which produced an interesting surface as can be seen in Fig. 2D. Next step of our study was focused on the elemental analysis of the AuPd NPs on the sensor surface by the EDS technique and the results are shown in Fig. 2E. Signature peaks related to the Au and Pd can be seen which guaranteed the successfulness of the electroception of the AuPd NPs onto the sensor surface. 
Fig. 2

\subsubsection{Optimization of the variables affecting the response of MIP/AuPd NPS/PDA/MWCNTs-CS-IL/GCE}

Response of a sensor is affected by some variables and to obtain a desired response, the optimal level of the variables must be determined. Therefore, in this section of our study we are going to optimize them. Phenolic group of the PDA is deprotonated at high $\mathrm{pHs}$ while its amino group is protonated at low $\mathrm{pH}$. Therefore, $\mathrm{pH}$ of the dopamine solution affected electropolymerization of the PDA and to optimize its effect, $\Delta I$ ( $\Delta I=I_{2}$ (peak current of the sensor after eluting template molecules)- $I_{1}$ (peak current of the sensor after adsorption of CHO molecules)) was investigated by differential pulse voltammetry (DPV). Variation of $\Delta I$ versus $\mathrm{pH}$ is shown in Fig. $3 \mathrm{~A} . \Delta I$ had the maximum value at $\mathrm{pH} 7$ therefore, it was selected as the optimal $\mathrm{pH}$ for electropolymerization of dopamine. Number of imprinted cavities is affected by the ratio of the CHO and ATP which was investigated to obtain optimized value of CHO/ATP and the results are shown in Fig. 3B. As can be seen, the maximum value of the $\Delta I$ was seen at $\mathrm{CHO} / \mathrm{ATP} \sim 2$ which was chosen as the optimal value for our next studies. Electropolymerization of the MIP onto the sensor surface was strongly affected by the number of scans of the CVs. Therefore, we investigated its effect on the $\Delta I$ and the results are shown in Fig. 3C. As can be seen, the desired response was observed at 6 cycles. According to the optimal levels of the variables investigated in this section, we will use them for our next studies in next sections.

Fig. 3

\subsubsection{Calibration of the MIP/AuPd NPs/PDA/MWCNTs-CS-IL/GCE response to CHO}

In this section, we were going to use EIS and DPV for calibration of the response of MIP/AuPd NPs/PDA/MWCNTs-CS-IL/GCE to CHO and to achieve this goal, EIS and DPV responses of the sensor versus increasing concentration of $\mathrm{CHO}$ were recorded and the results are shown in Fig. 4A and C. The DPV responses decreased with increasing concentration of $\mathrm{CHO}$ because of occupying the imprinted cavities on the MIP by $\mathrm{CHO}$ and hindering the electron transfer of $\left[\mathrm{Fe}(\mathrm{CN})_{6}\right]^{3-14-}$. The EIS responses were increased by increasing concentration of $\mathrm{CHO}$ because of blocking the cavities by $\mathrm{CHO}$ molecules and hindering charge transfer in some extent. In order to calibrate the EIS and DPV responses of MIP/AuPd NPs/PDA/MWCNTs-CS-IL/GCE with 
$\mathrm{CHO}$ concentration, its responses were regressed on $\mathrm{CHO}$ concentrations and the results are shown in Fig. 4B and D. As can be seen, EIS and DPV techniques could detect CHO in the range of 0.1-60 pM amd 1-50 pM, respectively. Sensitivity of the EIS and DPV techniques in determination of CHO were $488.21 \Omega \mathrm{pM}^{-1}$ and -3.112 $\mu \mathrm{A} \mathrm{pM}^{-1}$, respectively. Limits of detection $\left(\mathrm{LOD}=3 S_{\mathrm{b}} / m\right.$, where $S_{\mathrm{b}}$ is the standard deviation of the blank and $m$ is the slope of the calibration curve) of determination of CHO by EIS and DPV methods were $0.05 \mathrm{pM}$ and $0.2 \mathrm{pM}$, respectively.

\section{Fig. 4}

\subsubsection{Stability, repeatability, reproducibility and selectivity of the developed sensor}

Prior to the application of the developed sensor to the analysis of real samples, we have investigated some of its analytical characteristics and in this section, we describe them in some details. Stability of the sensor was investigated by weekly recording its EIS response to $20 \mathrm{pM} \mathrm{CHO} \mathrm{during} 7$ weeks and our records showed that the sensor was able to retain $94.5 \%$ of its original response which confirmed that the sensor response was stable. In order to examine repeatability of the sensor, it was applied to the determination of $20 \mathrm{pM} \mathrm{CHO}$ during one day by the EIS method and our records showed that a relative standard deviation $(R S D)$ of $2.33 \%$ was obtained which confirmed that the sensor response was repeatable. Reproducibility of the sensor response was examined by constructing six sensors and application of them to the determination of $20 \mathrm{pM} \mathrm{CHO}$. The results showed a $R S D$ of $3.1 \%$ was obtained which confirmed that the sensor response was reproducible. To investigate selectivity of the developed sensor, we have examined effect of several potential interference such as cholesterol, stigmasterol, estradiol, vitamin D3, testosterone, ascorbic acid, uric acid, progesterone, glucose, estrone and dopamine on the EIS response of MIP/AuPd NPs/PDA/MWCNTs-CS-IL/GCE to CHO and our results confirmed that the sensor response was only affected by high concentrations of vitamin D3 $(1 \mu \mathrm{M})$, progesterone $(0.1 \mu \mathrm{M})$ and cholesterol $(10 \mu \mathrm{M})$. The results confirmed that the sensor had higher selectivity for CHO than other interference which may be related to the good imprinting the polymer film. When the selectivity of the NIP modified electrode for determination of the $\mathrm{CHO}$ in the presence of the mentioned interference was examined, it didn't show any 
response which was related to the NIP film which hadn't any porosity and wasn't diffusible which hindered it to show any response.

\subsubsection{Application of the MIP/AuPd NPS/PDA/MWCNTS-CS-IL/GCE to the analysis of real samples}

In order to investigate the potential of the fabricated sensor for the analysis of the serum samples towards determination of $\mathrm{CHO}$ by the standard addition method, protein of the serum sample was removed by the addition of $\mathrm{HClO}_{4}$ and then, $2 \mathrm{~mL}$ of the serum was diluted to $20 \mathrm{~mL}$ by ethanol which was used to prepare five solutions containing different concentrations of CHO. Finally, the solutions were electrochemically (EIS) analyzed towards determination of $\mathrm{CHO}$ and the results are shown in Table 1. As can be seen, good recoveries were obtained which confirmed the successfulness of the developed sensor for the analysis of real samples towards determination of CHO.

\section{Table 1}

\section{Conclusions}

In this work, we have developed a novel, sensitive, selective and interesting MIES for determination of CHO in serum samples. This sensor has consisted of a bare GCE as its platform and several modifiers on its surface which have caused good operational abilities for the sensor. The MWCNTs-CS-IL has provided a conductive layer on which dopamine could electropolymerized to produce PDA which was able to act as bioinspired multifunctional modified materials to capture more template molecules. Bimetallic AuPd NPs acted as crosslinkers to form polymeric networks through $\mathrm{Au}-\mathrm{S}$ between $\mathrm{Au}$ and the functional monomer ATP. PDA films have metal binding ability to stabilize AuNPs and can form intermolecular forces between ATP and PDA. Detection of the CHO molecules was based on decreasing and increasing DPV and EIS response of the sensor, respectively, with increasing concentration of $\mathrm{CHO}$ which was related to the occupying cavities on the MIP by $\mathrm{CHO}$ molecules and hindering the electron transfer of $\left[\mathrm{Fe}(\mathrm{CN})_{6}\right]^{3-/ 4-}$. On the whole, selection of a good structure for the MIES in this work helped us to develop a sensitive, selective, repeatable and reproducible method for determination of $\mathrm{CHO}$ in real samples.

\section{Acknowledgments}




\section{Medical Sciences.}

\section{References}

[1] J.H. Menkes, J.R. Schimshock, P.D. Swanson, Cerebrotendinous xanthomatosis. The storage of cholestanol within the nervous system, Arch. Neurol. 19 (1968) 47-53.

[2] G. Salen, Cholestanol deposition in cerebrotendinous xanthomatosis. A possible mechanism, Ann. Intern. Med. 75 (1971) 843-851.

[3] J. Koopman, J.C. van der Molen, B.G. Wolthers, A.E.J. de Jager, R.J. Waterreus, C.H. Gips, Capillary gas chromatographic determination of cholestanol/cholesterol ratio in biological fluids. Its potential usefulness for the follow-up of some liver diseases and its lack of specificity in diagnosing CTX (cerebrotendinous xanthomatosis), Clin. Chim. Acta 137 (1984) 305-315.

[4] K. Nikkilae, T.A. Miettinen, Serum Cholesterol Precursors, Cholestanol, and Plant Sterols in Primary Biliary Cirrhosis, Scand. J. Gastroenterol. 23 (1988) 967-972.

[5] K. Nikkilae, Liver transplantation restores low serum levels of very low density and high density lipoproteins in end-stage primary biliary cirrhosis, Ann. Med. 24 (1992) 129-136.

[6] K. Nikkilae, K. Hockerstedt, T.A. Miettinen, Serum and hepatic cholestanol, squalene and noncholesterol sterols in man: a study on liver transplantation, Hepatology 15 (1992) 863-870.

[7] G. Salen, S.M. Grundy, The metabolism of cholestanol, cholesterol, and bile acids in cerebrotendinous xanthomatosis, J. Clin. Invest. 52 (1973) 2822-2835.

[8] T.T. Ishikawa, J.B. Brazier, L.E. Stewart, R.W. Fallot, C.J. Glueck, Direct quantitation of cholestanol in plasma by gas-liquid chromatography, J. Lab. Clin. Med. 87 (1976) 345-353.

[9] G. Salen,V. Berginer,V. Shore, I. Horak, E. Horak, G.S. Tint, S. Shefer, Increased concentrations of cholestanol and apolipoprotein B in the cerebrospinal fluid of patients with cerebrotendinous xanthomatosis. Effect of chenodeoxycholic acid, New Engl. J. Med. 316 (1987) 1233-1238.

[10] Y. Seyama, K. Ichikawa, T. Yamakawa, Quantitative determination of cholestanol in plasma with mass fragmentography. Biochemical diagnosis of cerebrotendinous xanthomatosis, J. Biochem. 80 (1976) 223-228.

[11] C. Matsuoka, H. Nohta, N. Kuroda, Y. Ohkura, Simultaneous determination of cholestanol and cholesterol in human serum by high-performance liquid chromatography with fluorescence detection, J. Chroma- togr. 341 (1985) 432-436.

[12] T. Iwata, M. Yamaguchi, M. Nakamura, Highly sensitive and simple determination of cholesterol and cholestanol in human serum by high-performance liquid chromatography with fluorescence detection, J. Chromatogr. 421 (1987) 43-50.

[13] Y. Tsuruta, T. Teranishi, Y. Date, K. Kohashi, Simultaneous determination of cholesterol and cholestanol in human serum by high-performance liquid chromatography using 3-(5,6-methylenedioxy-2-phthalimidyl)benzoyl azide as precolumn fluorescent labelling reagent, J. Chromatogr. 617 (1993) 213-220.

[14] T. Kasama, D.-S. Byun, Y. Seyama, Quantitative analysis of sterols in serum by high-performance liquid chromatography. Application to the biochemical diagnosis of cerebrotendinous xanthomatosis, J. Chromatogr. 400 (1987) 241-246. 
[15] K.S. Kim, K. Kano, T. Kasama, Y. Ishii, H. Yamashita, Y. Seyama, Effects of cholestanol feeding on corneal dystrophy in mice, Biochim. Biophys. Acta 1085 (1991) 343-249.

[16] M. Kuriyama, J. Fujiyama, T. Kasama, M. Osame, High levels of plant sterols and cholesterol precursors in cerebrotendinous xanthomatosis, J. Lipid Res. 32 (1991) 223-229.

[17] H. Hideka, T. Nakamura, T. Aosi, H. Kojima, Y. Nakajima, K. Kosugi, I. Hatanaka, M. Harada, M. Kobayashi, A. Tamura, T. Fujii, Y. Shigeta, Increased plasma plant sterol levels in heterozygotes with sitosterolemia and xanthomatosis, J. Lipid Res. 31 (1990) 881-888.

[18] M. Riskin, R. Tel-Vered, T. Bourenko, E. Granot, Imprinting of molecular recognition sites through electropolymerization of functionalized au nanoparticles: Development of an electrochemical tnt sensor based on $\pi$-donor-acceptor interactions, JACS 130 (2008) 9726-9733.

[19] Z. Altintas, M. Gittens, A. Guerreiro, K.A. Thompson, J. Walker, S. Piletsky, Detection of waterborne viruses using high affinity molecularly imprinted polymers, Anal. Chem. 87 (2015) 6801-6807.

[20] V. Suryanarayanan, C.T. Wu, K.C. Ho, Molecularly Imprinted Electrochemical Sensors, Electroanalysis, 22 (2010) 1795-1811.

[21] J. Ji, Z. Zhou, X. Zhao, J. Sun, X. Sun, Electrochemical sensor based on molecularly imprinted film at Au nanoparticles-carbon nanotubes modified electrode for determination of cholesterol, Biosens. Bioelectron. 66 (2015) 590-595.

[22] F.T.C. Moreira, R.A.F. Dutra, J.P.C. Noronha, M.G.F. Sales, Myoglobin-biomimetic electroactive materials made by surface molecular imprinting on silica beads and their use as ionophores in polymeric membranes for potentiometric transduction, Biosens. Bioelectron. 26 (2011) 4760-4766.

[23] N. Perez, M.J. Whitcombe, E.N. Vulfson, Surface Imprinting of Cholesterol on Submicrometer Core-Shell Emulsion Particles, Macromolecules 34 (2001.) 830-836.

[24] H.F. Wang, Y. He, T.R. Ji, X.P. Yan, Surface Molecular Imprinting on Mn-Doped ZnS Quantum Dots for Room-Temperature Phosphorescence Optosensing of Pentachlorophenol in Water, Anal. Chem. 81 (2009.) 16151621.

[25] Y.T. Wang, Y.X. Zhou, J. Sokolov, B. Rigas, K. Levon, M. Rafailovich, A potentiometric protein sensor built with surface molecular imprinting method, Biosens. Bioelectron. 24 (2008) 162-166.

[26] H. Lee, S.M. Dellatore, W.M. Miller, P.B. Messersmith, Mussel-inspired surface chemistry for multifunctional coatings, Science 318 (2007) 426-430.

[27] M.J. LaVoie, B.L. Ostaszewski, A. Weihofen, M.G. Scholssmacher, D.J. Selkoe, Dopamine covalently modifies and functionally inactivates parkin, Nat. Med. 11 (2005) 1214-1221.

[28] C.G. Pierpont, C.W. Lange, The chemistry of transition metal complexes containing catechol and semiquinone ligands, Prog. Inorg. Chem. 41 (1994) 331-442.

[29] B. Rezaei, N. Majidi, A.A. Ensafi, H. Karimi-Maleh, Molecularly imprinted-multiwall carbon nanotube paste electrode as a biosensor for voltammetric detection of rutin, Anal. Meth. 3 (2011) 2510-2516.

[30] M.C. Buzzeo, R.G. Evans, R.G. Compton, Non-haloaluminate room-temperature ionic liquids in electrochemistry-a review, Chem. Phys. Chem. 5 (2004) 1106-1120.

[31] N. Maleki, A. Safavi, F. Tajabadi, High-performance carbon composite electrode based on an ionic liquid as a binder, Anal. Chem. 78 (2006) 3820-3826.

[32] M. Zhang, A. Smith, W. Gorski, Carbon nanotube-chitosan system for electrochemical sensing based on dehydrogenase enzymes, Anal. Chem. 76 (2004) 5045-5050.

[33] X. Lu, Z. Wen, J. Lin, Hydroxyl-containing antimony oxide bromide nanorods combined with chitosan for biosensors, Biomaterials 27 (2006) 5740-5747.

[34] J. Lin, W. Qu, S. Zhang, Disposable biosensor based on enzyme immobilized on Au-chitosan-modified indium tin oxide electrode with flow injection amperometric analysis, Anal. Biochem. 360 (2007) 288 -293.

[35] E. Katz, I. Willner, J. Wang, Electroanalytical and bioelectroanalytical systems based on metal and semiconductor nanoparticles, Electroanalysis 16 (2004) 19-44.

[36] R. Khodarahmi, S. Khateri, H. Adibi, V. Nasirian, M. Hedayati, E. Faramarzi, S. Soleimani, HC. Goicoechea, A.R. Jalalvand, Chemometrical-electrochemical investigation for comparing inhibitory effects of quercetin and its 
sulfonamide derivative on human carbonic anhydrase II: Theoretical and experimental evidence, Int. J. Biol. Macromol. 136 (2019) 377-385.

[37] A.R. Jalalvand, A. Haseli, F. Farzadfar, H.C. Goicoecheac, Fabrication of a novel biosensor for biosensing of bisphenol A and detection of its damage to DNA, Talanta 201 (2019) 350-357.

[38] A.R. Jalalvand, Fabrication of a novel and ultrasensitive label-free electrochemical aptasensor for detection of biomarker prostate specific antigen, Int. J. Biol. Macromol. 126 (2019) 1065-1073.

[39] M.M. Zangeneh, H. Norouzi, M. Mahmoudi, H.C. Goicoechea, A.R. Jalalvand, Fabrication of a novel impedimetric biosensor for label free detection of DNA damage induced by doxorubicin, Int. J. Biol. Macromol. 124 (2019) 963-971.

[40] A.R. Jalalvand, H.C. Goicoechea, H.-W. Gu, An interesting strategy devoted to fabrication of a novel and high performance amperometric sodium dithionite sensor, Microchem. J. 144 (2019) 6-12.

[41] A.R. Jalalvand, M.B. Gholivand, H.C. Goicoechea, T. Skov, K. Mansouri, Mimicking enzymatic effects of cytochrome P450 by an efficient biosensor for in vitro detection of DNA damage, Int. J. Biol. Macromol. 79 (2015) 1004-1010.

[42] M.B. Gholivand, A.R. Jalalvand, H.C. Goicoechea, G. Paimard, T. Skov, Surface exploration of a roomtemperature ionic liquid-chitin composite film decorated with electrochemically deposited PdFeNi trimetallic alloy nanoparticles by pattern recognition: an elegant approach to developing a novel biotin biosensor, Talanta 131 (2015) 249-258.

[43] M.B. Gholivand, A.R. Jalalvand, H.C. Goicoechea, Computer-assisted electrochemical fabrication of a highly selective and sensitive amperometric nitrite sensor based on surface decoration of electrochemically reduced graphene oxide nanosheets with CoNi bimetallic alloy nanoparticles, Mater. Sci. Eng. C 40 (2014) 109120.

[44] G. Mohammadi, E. Faramarzi, M. Mahmoudi, S. Ghobadi, A.R. Ghiasvand, H.C. Goicoechea, A.R. Jalalvand, Chemometrics-assisted investigation of interactions of Tasmar with human serum albumin at a glassy carbon disk: application to electrochemical biosensing of electro-inactive serum albumin, J. Pharm. Biomed. Anal. 156 (2018) 23-35.

[45] M.B. Gholivand, A.R. Jalalvand, H.C. Goicoechea, M. Omidi, Investigation of interaction of nuclear fast red with human serum albumin by experimental and computational approaches, Spectrochim. Acta A 115 (2013) $516-527$.

[46] A.R. Jalalvand, S. Ghobadi, H.C. Goicoechea, H.W. Gu, E. Sanchooli, Investigation of interactions of Comtan with human serum albumin by mathematically modeled voltammetric data: A study from bio-interaction to biosensing, Bioelectrochemistry 123 (2018) 162-172.

[47] M.B. Gholivand, A.R. Jalalvand, H.C. Goicoechea, Multivariate analysis for resolving interactions of carbidopa with dsDNA at a fullerene-C $60 / G C E$, Int. J. Biol. Macromol. 69 (2014) 369-381.

[48] M.B. Gholivand, A.R. Jalalvand, H.C. Goicoechea, R. Gargallo, T. Skov, Chemometrics: An important tool for monitoring interactions of vitamin B7 with bovine serum albumin with the aim of developing an efficient biosensing system for the analysis of protein, Talanta 132 (2015) 354-365.

[49] A.R. Jalalvand, S. Ghobadi, V. Akbari, H.C. Goicoechea, E. Faramarzi, M. Mahmoudi, Mathematical modeling of interactions of cabergoline with human serum albumin for biosensing of human serum albumin, Sens. Bio-Sens. Research 25 (2019) 100297.

[50] A.R. Jalalvand, S. Ghobadi, H.C. Goicoechea, E. Faramarzi, M. Mahmoudi, Matrix augmentation as an efficient method for resolving interaction of bromocriptine with human serum albumin: Trouble shooting and simultaneous resolution, Heliyon 5 (2019) e02153.

[51] K. Ghanbari, M. Roushani, F. Farzadfar, H.C. Goicoechea, A.R. Jalalvand, Developing a four-dimensional voltammetry as a powerful electroanalytical methodology for simultaneous determination of three colorants in the presence of an uncalibrated interference, Chemom. Intell. Lab. Syst. 189 (2019) 27-38.

[52] A.R. Jalalvand, H.C. Goicoechea, D.N. Rutledge, Applications and challenges of multi-way calibration in electrochemical analysis, TrAC Trends Anal. Chem. 87 (2017) 32-48. 
[53] A.R. Jalalvand, H.C. Goicoechea, Applications of electrochemical data analysis by multivariate curve resolution-alternating least squares, TrAC Trends Anal. Chem. 88C (2017) 134-166.

[54] A.R. Jalalvand, M. Roushani, H.C. Goicoechea, D.N. Rutledge, H.W. Gu, MATLAB in electrochemistry: A review, Talanta 194 (2019) 205-225. 


\section{Caption to figures:}

Fig. 1. (A) and (B) CVs and EISs of different electrodes recorded in the probe solution consisting of $5 \mathrm{mM}$ $\mathrm{K}_{3} \mathrm{Fe}(\mathrm{CN})_{6}$ and $5 \mathrm{mM} \mathrm{K} \mathrm{K}_{4} \mathrm{Fe}(\mathrm{CN})_{6}$ prepared in $0.1 \mathrm{M} \mathrm{KCl}$ : (a) GCE, (b) MWCNTs-CS-IL/GCE, (c) PDA/MWCNTs-CS-IL/GCE, (d) AuPd NPs/PDA/MWCNTs-CS-IL/GCE, (e) MIP/AuPd NPs/PDA/MWCNTsCS-IL/GCE and (f) NIP/AuPd NPs/PDA/MWCNTs-CS-IL/GCE.

Fig. 2. (A)-(D) SEM images recorded from the surface of MWCNTs-CS-IL/GCE, PDA/MWCNTs-CS-IL/GCE, AuPd NPs/PDA/MWCNTs-CS-IL/GCE, MIP/AuPd NPs/PDA/MWCNTs-CS-IL/GCE, respectively. (E) elemental analysis by the EDS and signature peaks related to the $\mathrm{Au}$ and $\mathrm{Pd}$.

Fig. 3. Investigation and optimization of the effects of (A) $\mathrm{pH}$, (B) CHO/ATP and (C) number of CV cycles on the sensor response.

Fig. 4. (A) EIS responses of MIP/AuPd NPs/PDA/MWCNTs-CS-IL/GCE to different concenrtions of CHO ranging in 0.1-60 pM, (B) regresion of the EIS responses of the senor on CHO concetrations, (C) EIS responses of MIP/AuPd NPs/PDA/MWCNTs-CS-IL/GCE to different concentions of CHO ranging in 1-50 pM and (D) regresion of the DPV responses of the senor on $\mathrm{CHO}$ concetrations.

Scheme 1. Schematic representation of the procedure developed in this work for determination of CHO. 


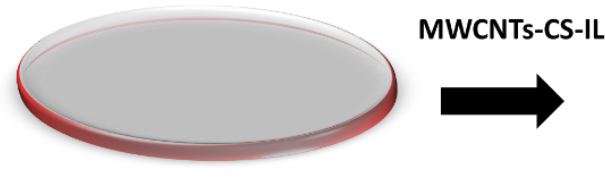

GCE

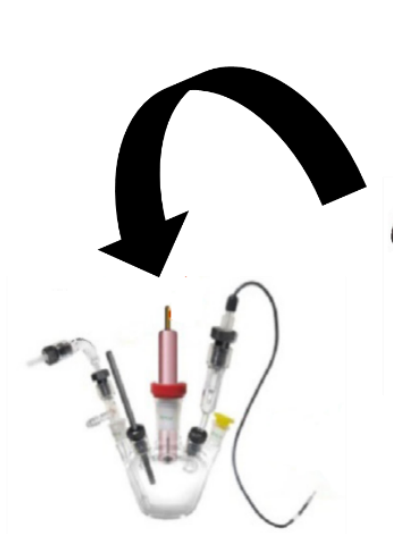

Electrochemical sensing of $\mathrm{CHO}$

Working Electrode

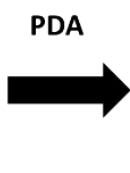

MWCNTS-CS-IL/GCE
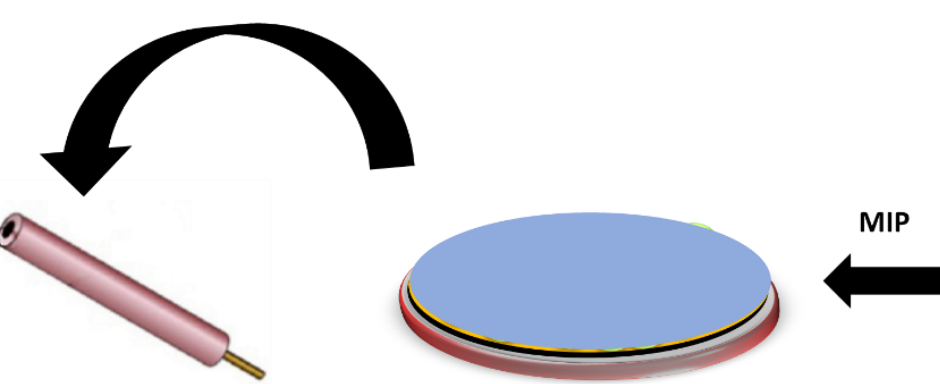

MIP/AuPd NPs/PDA/MWCNTs-CS-IL/GCE

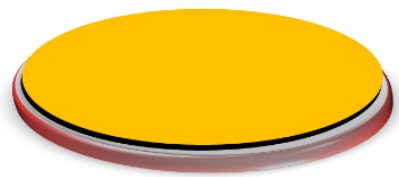

PDA/MWCNTS-CS-IL/GCE
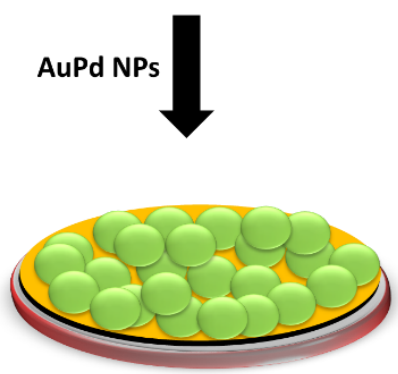

AuPd NPs/PDA/MWCNTs-CS-IL/GCE

Scheme 1. 
Table 1. Application of the developed sensor to the analysis of real samples for determination of CHO.

\begin{tabular}{cccc}
\hline Sample & Added $(\mathrm{pM})$ & Found $(\mathrm{pM})$ & Recovery $(\%)$ \\
\hline 1 & 15.0 & 14.8 & 98.7 \\
2 & 35.0 & 35.9 & 102.5 \\
3 & 10.0 & 9.8 & 98.0 \\
4 & 28.0 & 29.0 & 103.4 \\
5 & 42.0 & 40.5 & 96.4 \\
\hline
\end{tabular}



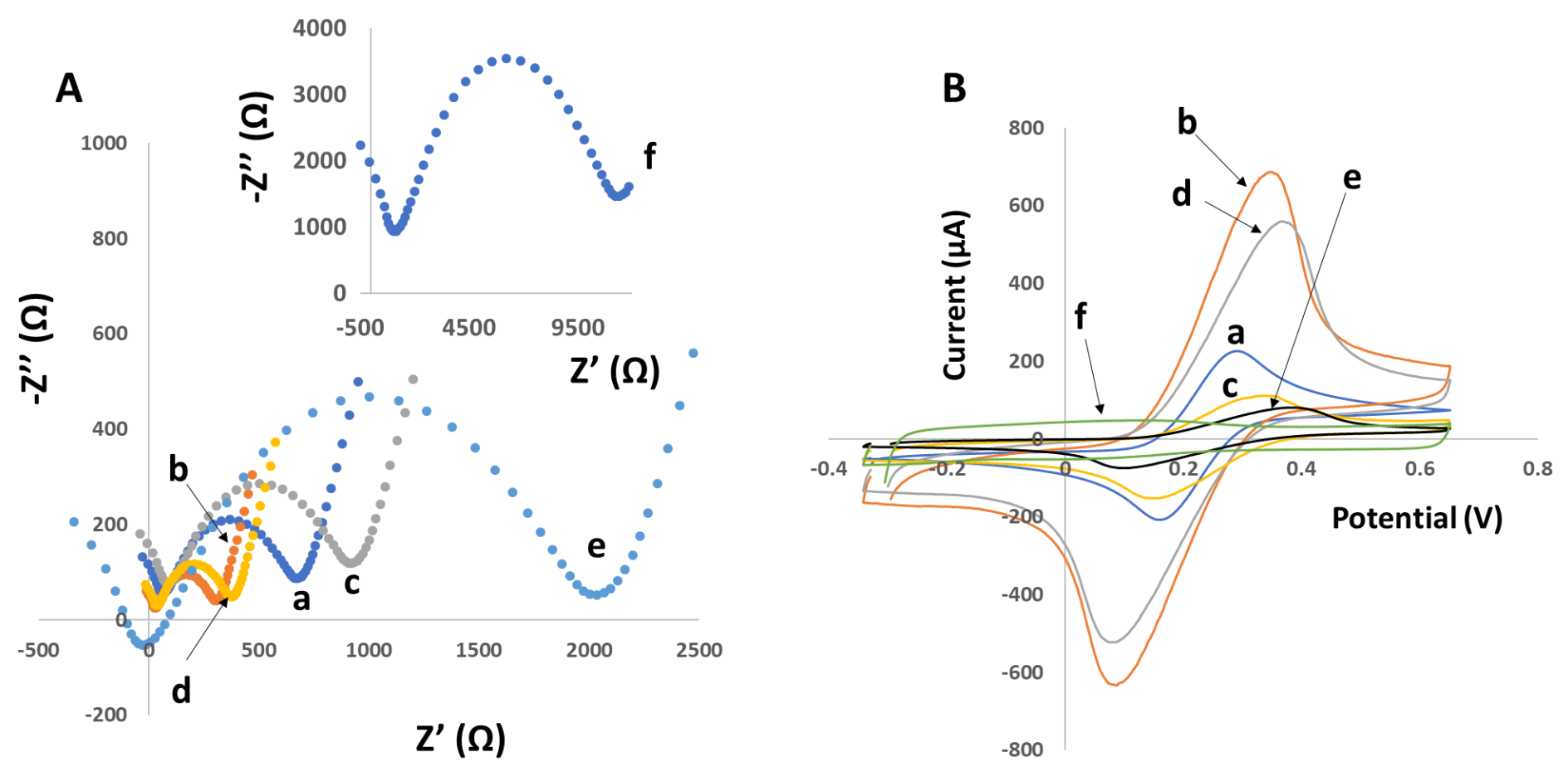

Fig. 1. 

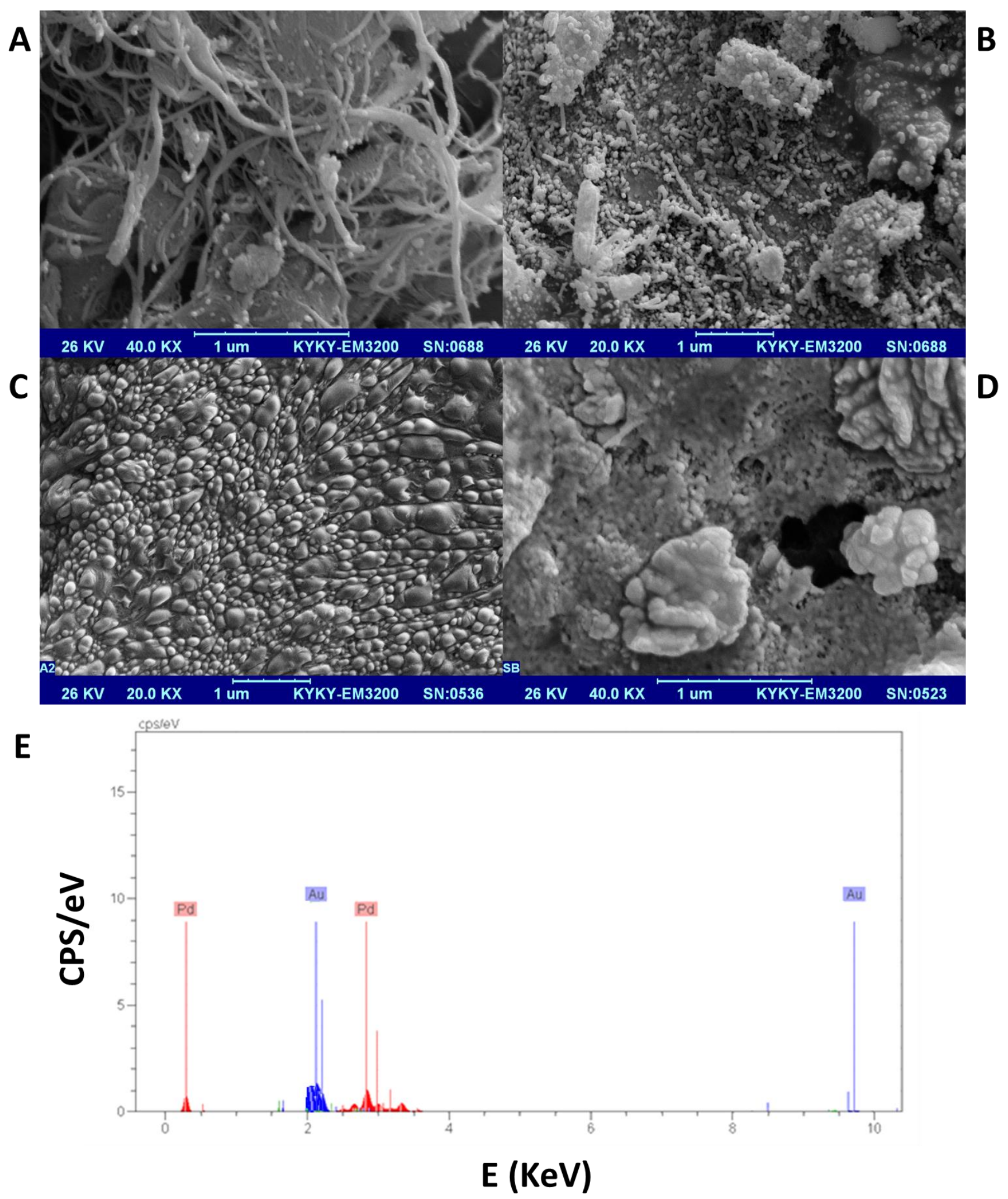

Fig. 2. 
A

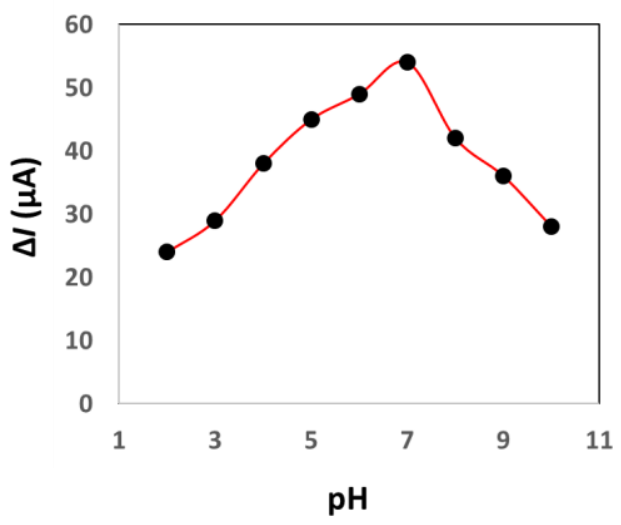

B

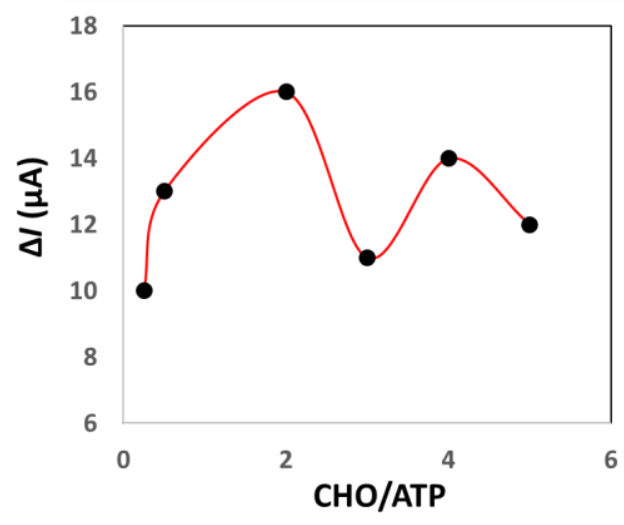

\section{C}

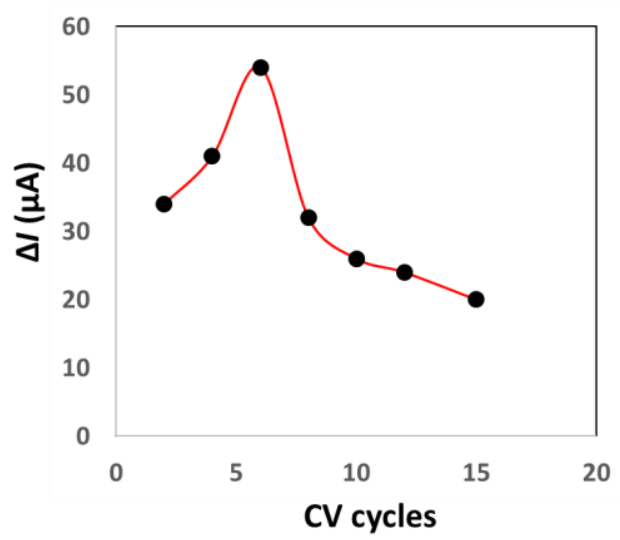

Fig. 3. 
A
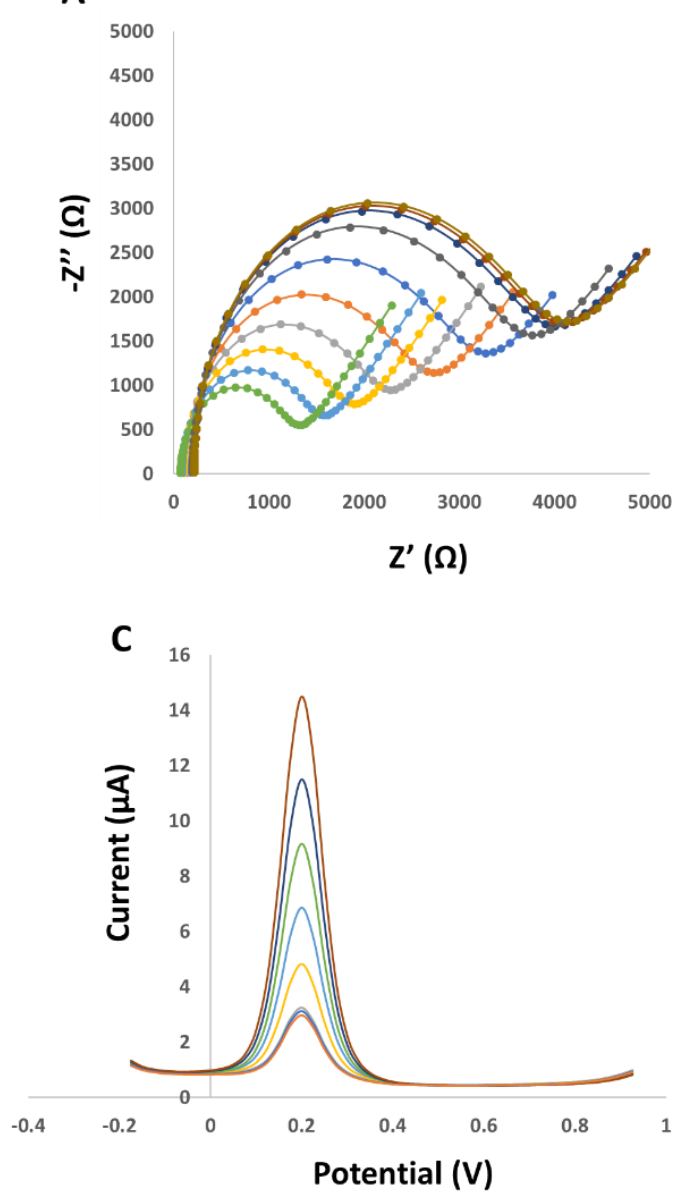

B
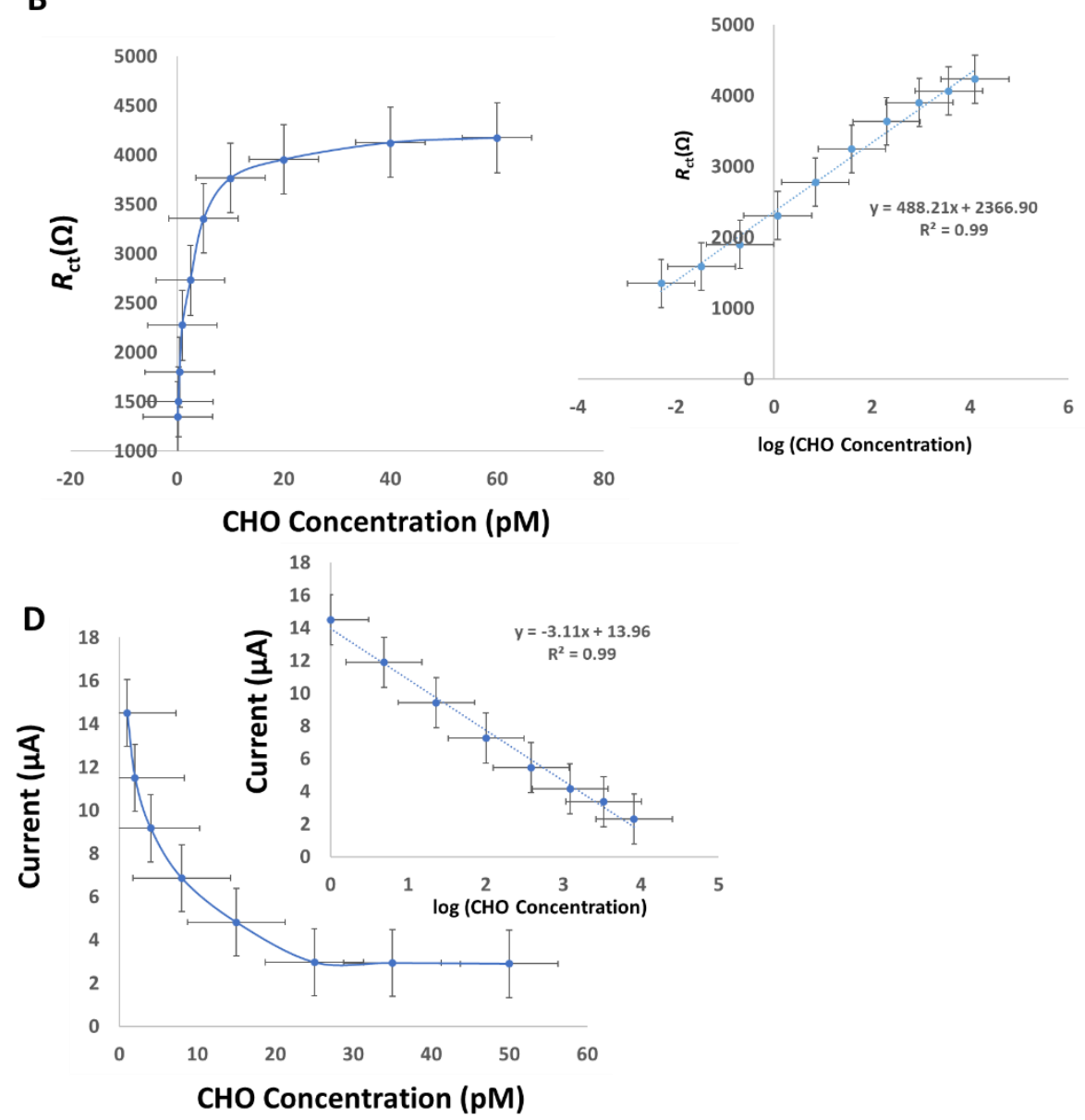

Fig. 4. 